\title{
Centre d'évaluation des dispositifs médicaux de Montpellier (CED2M). Applications en laboratoire et en clinique
}

\section{RÉSUMÉ}

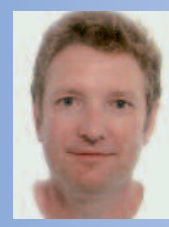

Jean-Christophe EGEA

MCU-PH

Laboratoire Biosanté et Nanoscience, EA 4203,

Faculté d'Odontologie,

Université Montpellier 1 ,

1840 boulevard de la Liberté,

34830 Clapiers.

\section{Bruno PELISSIER}

MCU-PH,

Laboratoire Biosanté et Nanoscience, EA 4203,

Faculté d'Odontologie,

Université Montpellier 1.

\section{Alban DESOUTTER}

Attaché de Recherche clinique,

Laboratoire Biosanté et Nanoscience, EA 4203.

Faculté d'Odontologie,

Université Montpellier 1.

\section{Marie-Alix FAUROUX}

Ancien AHU,

Laboratoire Biosanté et Nanoscience, EA 4203,

Faculté d'Odontologie,

Université Montpellier 1.

\section{Laurence POURREYRON}

\section{MCU-PH,}

Laboratoire Biosanté et Nanoscience, EA 4203,

Faculté d'Odontologie,

Université Montpellier 1.

\section{Frédéric CUISINIER}

PU-PH ,

Laboratoire Biosanté et Nanoscience, EA 4203,

Faculté d'Odontologie,

Université Montpellier 1.

\section{Bruno JACQUOT}

MCU-PH,

Laboratoire Biosanté et Nanoscience, EA 4203,

Faculté d'Odontologie,

Université Montpellier 1.

La directive européenne 2007/47/CE est d'application obligatoire depuis le 21 mars 2010 pour l'ensemble de I'Union européenne. Elle impose maintenant une évaluation clinique pour tous les dispositifs médicaux avec un renforcement en ce qui concerne la sécurité et la performance.

Pour satisfaire à ce besoin d'évaluation, le laboratoire Biosanté et Nanoscience a créé le Centre d'évaluation des dispositifs médicaux de Montpellier (CED2M).

Deux évaluations sont présentées, effectuées dans le cadre de CED2M.

La première est réalisée en laboratoire sur un prototype de lampe à polymériser de type LED. Trois paramètres standards corrélés à la photopolymérisation ont été évalués pour les applications en dentisterie restauratrice et un test de résistance au cisaillement a été effectué pour les matériaux de collage utilisés en orthodontie.

La seconde est en cours de réalisation, dans le service d'odontologie du CHU de Montpellier sur un nouvel implant en zircone. Le protocole de l'étude clinique est décrit.

- dispositif médical

- directive 93/42/CEE

- photo-polymérisation

- implant dentaire
AOS 2012;259:219-230

DOI: 10.1051/aos/2012303

C EDP Sciences 
$>$

Un dispositif médical est un instrument, appareil, équipement ou encore un logiciel destiné, par son fabricant, à être utilisé chez I'homme à des fins, notamment, de diagnostic, de prévention, de contrôle, de traitement, d'atténuation d'une maladie ou d'une blessure. L'action principale d'un dispositif médical n'est pas obtenue par des moyens pharmacologique ou immunologique ni par métabolisme, elle est essentiellement mécanique (directive 93/42/CEE [1]). Pour les dispositifs médicaux, I'Union européenne constitue un marché unique régi par les mêmes règles dans chacun des Etats membres.

La directive européenne 2007/47/CE, entrée en vigueur le 11 octobre 2007, est d'application obligatoire depuis le 21 mars 2010 pour l'ensemble de I'Union européenne. L'impact de la directive n'est pas négligeable pour les industriels. En effet, elle impose maintenant une évaluation clinique pour tous les dispositifs médicaux, quelle que soit leur classe, évaluation qui doit par ailleurs être renforcée en ce qui concerne la sécurité et la performance. Enfin, le suivi clinique devra être poursuivi durant tout le cycle de vie du dispositif médical, et ce, même après le marquage CE.

Les dispositifs médicaux sont répartis en quatre classes (I, Ila, IIb, III), correspondant à des niveaux de risque croissants de I à III. Les prothèses dentaires (amovibles ou fixes) fabriquées spécifiquement pour un patient déterminé sont considérées comme des dispositifs médicaux sur mesure. Les dispositifs de la classe I peuvent se satisfaire d'une auto-certification par le fabricant lui-même, aboutissant au marquage CE. Pour les autres classes, une intervention d'un organisme notifié est obligatoire, avant que le marquage $C E$ ne puisse être apposé sur le dispositif.

Pour satisfaire à ce besoin d'évaluation, le laboratoire Biosanté et Nanoscience a créé le Centre d'évaluation des dispositifs médicaux de Montpellier (CED2M) qui associe deux structures complémentaires.

Une première structure permet de confirmer la sécurité et l'efficacité des dispositifs médicaux testés et d'accéder si nécessaire aux études cliniques. Des tests de laboratoire de mesure des propriétés physicochimiques et mécaniques sont associés à différentes analyses chimiques et techniques d'imagerie.

Une seconde structure permet de concevoir et de réaliser les études cliniques adaptées aux différents dispositifs médicaux. Des spécialistes des différents domaines odontologiques travaillent en permanence sur de nouveaux projets scientifiques. Un Attaché de recherche clinique dédié s'occupe de la rédaction et du dépôt des dossiers, du suivi des études et de la collecte des données.

Nous présentons deux évaluations, parmi d'autres, effectuées dans le cadre de CED2M. La première est réalisée en laboratoire sur un prototype de lampe à polymériser. La seconde est réalisée en clinique dans le service d'odontologie du CHU de Montpellier sur un nouvel implant en zircone.

\section{Évaluation au laboratoire d'un prototype de lampe à polymériser}

Les lampes à photopolymériser sont répertoriées en classe I dans la classification des dispositifs médicaux.
Le but de cette étude a été d'évaluer un prototype d'un nouveau modèle de lampe à photopolymériser, basé sur la technologie LED, pour 
des applications en dentisterie restauratrice et en orthodontie et proposée par la société Acteon. La lampe testée avant mise sur le marché est dénommée Scanwave by MiniLED ${ }^{\circledR}$ (fig. 1), marque déposée le 5 janvier 2011.

Ce produit dispose de plusieurs types de diodes pour élargir son spectre d'émission sans élever globalement la puissance émise. En effet, une attention particulière est portée par le fabricant à la réduction des phénomènes d'élévation thermiques délétères pour les différents tissus dentaires [2] et oraux et désagréables pour les patients.

Pour tester la validité du concept et définir les modes de polymérisation les plus adaptés aux différentes situations cliniques rencontrées en dentisterie restauratrice et en orthodontie, deux études ont été conduites en parallèle par les universités de Montpellier et de Birmingham.

Pour l'université de Montpellier, dans le cadre de CED2M, trois paramètres standards corrélés à la polymérisation photonique ont été évalués pour les applications en dentisterie restauratrice :
- l'augmentation de température ;

- le retrait de polymérisation ;

- le degré de conversion.

Pour les applications en orthodontie, l'efficacité de la photopolymérisation pour le collage de boîtiers orthodontiques par l'intermédiaire de ciments et colles orthodontiques a été évaluée par un test de résistance au cisaillement.

\section{Les lampes \\ à photopolymériser testées}

Le prototype Scanwave by MiniLED ${ }^{\circledR}$ possède un large spectre d'émission de 390 à $510 \mathrm{~nm}$ et un scan automatique des longueurs d'onde couvrant les différents photo-initiateurs (fig. 2) utilisés sur le marché (CamphroQuinone [CQ], Lucirin ${ }^{\circledR T P O}$ [TPO] et PhénylPropaneDione [PPD]).

Les sources lumineuses LED de la lampe Scanwave sont centrées sur 410 nm, 440 nm, $460 \mathrm{~nm}$ et $480 \mathrm{~nm}$. La conception du bloc optique donne une configuration électrique de
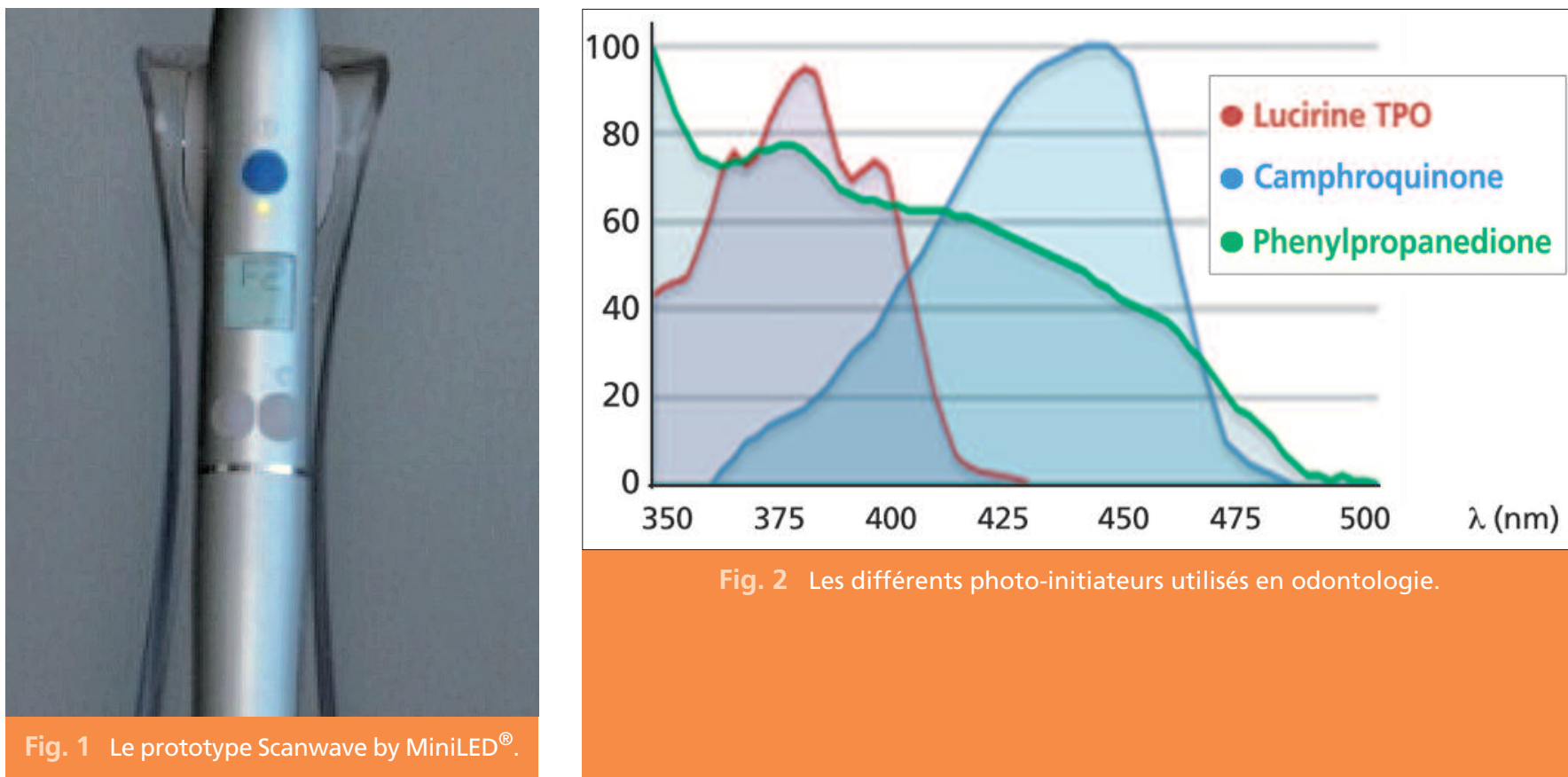

Fig. 2 Les différents photo-initiateurs utilisés en odontologie. 
trois branches de LED, précédées d'une LED de tête. Le concept novateur de Scanwave réalise un balayage des photo-initiateurs. Scanwave combine aussi les fonctions traditionnelles de Satelec ${ }^{\circledR}$ comme le boitier en aluminium évitant tout risque de hausse de la température et la visée laser qui assure le positionnement précis de l'intensité lumineuse.

La lampe Scanwave a été comparée à deux lampes LED de référence du marché :

- la lampe Valo ${ }^{\circledR}$ de Ultradent.

- la lampe Bluephase ${ }^{\circledR} 20$ i de Ivoclar Vivadent.

Le composite testé est Evoceram ${ }^{\circledR}$ de IvoclarVivadent.

\section{> Lampe Scanwave ${ }^{\circledR}$}

- Gamme de longueurs d'onde : 390 à $510 \mathrm{~nm}$.

- Modes de polymérisation

- Mode Full scan (affichage F1) avec activation TPO+PPD+CQ (Pulsé 9 x 1s ou Continu 9s).

- Mode Full scan inverse (affichage F2) (Pulsé $9 \times 1$ s ou Continu 9s).

- Mode TPO (affichage tP) avec activation des LED 440 et 410 nm (Pulsé $9 \times 1$ s ou Continu 9s).

- Mode PPD (affichage PP) avec activation des LED 440 et 460 nm (Pulsé $9 \times 1$ s ou Continu 9s).

- Mode CQ (affichage Co) avec activation des LED 440 et 480 nm (Pulsé $9 \times 1$ s ou Continu 9s).

- Mode Scan Séquentiel (affichage SS) avec activation séquence TPO PPD CQ (Continu 9s).

$>$ Lampe Valo ${ }^{\circledR}$

- Gamme de longueurs d'onde : 395 à 480 nm.

- Modes de polymérisation

- Mode Standard - $1000 \mathrm{~mW} / \mathrm{cm}^{2}$ (5 / 10 / 15 / 20 secondes).

- Mode High Power - $1400 \mathrm{~mW} / \mathrm{cm}^{2}$ (1 / $2 / 3$ / 4 secondes).

- Mode Xtra Power - 4500 mW/cm² (3 secondes).

\section{> Lampe Bluephase ${ }^{\circledR} 20 i$}

- Gamme de longueurs d'onde : 380 à 515 nm.

- Modes de polymérisation

- Mode Low Power - $650 \mathrm{~mW} / \mathrm{cm}^{2}$ (10 à 30 secondes).

- Mode Soft Start - 650/1200 mW/cm² (10 à 30 secondes).

- Mode High Power - $1400 \mathrm{~mW} / \mathrm{cm}^{2}$ (10 à 30 secondes).

- Mode Turbo - $4500 \mathrm{~mW} / \mathrm{cm}^{2}$ (3 à 5 secondes). Pour Acteon, la comparaison de Scanwave avec deux lampes de référence du marché avait pour intérêt de valider leur concept original. Mais une comparaison directe était impossible, les modes de polymérisation des différentes lampes donnant des irradiations différentes en intensité et en temps et non superposables. Aussi, seuls les résultats obtenus avec la lampe Scanwave sont donnés dans cet article.

\section{Détermination de l'élévation de température durant l'insolation}

Les tests sont réalisés par l'intermédiaire d'un thermocouple de type $K$, associé à un thermomètre digital (Testo), dans un moule de téflon spécifique, selon un montage réalisé au laboratoire. Ce montage correspond aux études réalisées sur le sujet dans la littérature $[3,4]$.

Le thermocouple (haute performance) a été spécifiquement réalisé par le Laboratoire des Verres de Montpellier (UMR 5587). Le moule en téflon a été réalisé par la société Isoflon (dimensions $6 \mathrm{~mm}$ de diamètre et $2 \mathrm{~mm}$ de hauteur). Le composite Evoceram ${ }^{\circledR}$ condensé dans le moule sous un strip de mylar est placé de manière reproductible à $5 \mathrm{~mm}$ du thermocouple. 
Les échantillons sont insolés à température ambiante.

Une première mesure est effectuée durant la première insolation qui correspond à la réaction exothermique de prise associée à la chaleur générée par l'irradiation. Une seconde insolation est effectuée après retour à l'ambiante, dont la mesure correspond à la chaleur de l'ir- radiation. Les différentes mesures sont réalisées au travers du même composite polymérisé (fig. 3 a et b).

La lampe Scanwave démontre en moyenne une élévation de température dans une fourchette basse parmi les différentes lampes testées. Le mode TP qui associe les LED 410 et $440 \mathrm{~nm}$ démontre la plus faible élévation de tempéra-

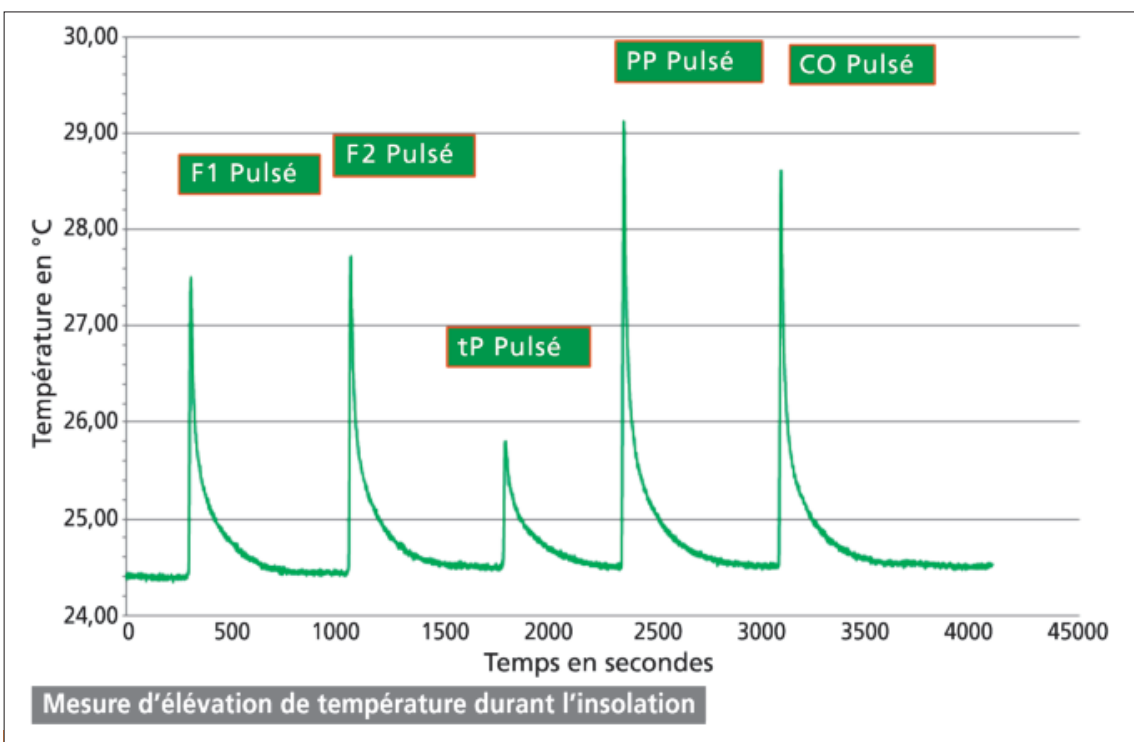

Fig. 3 a Courbes d'élévation de température du prototype Scanwave en mode pulsé.

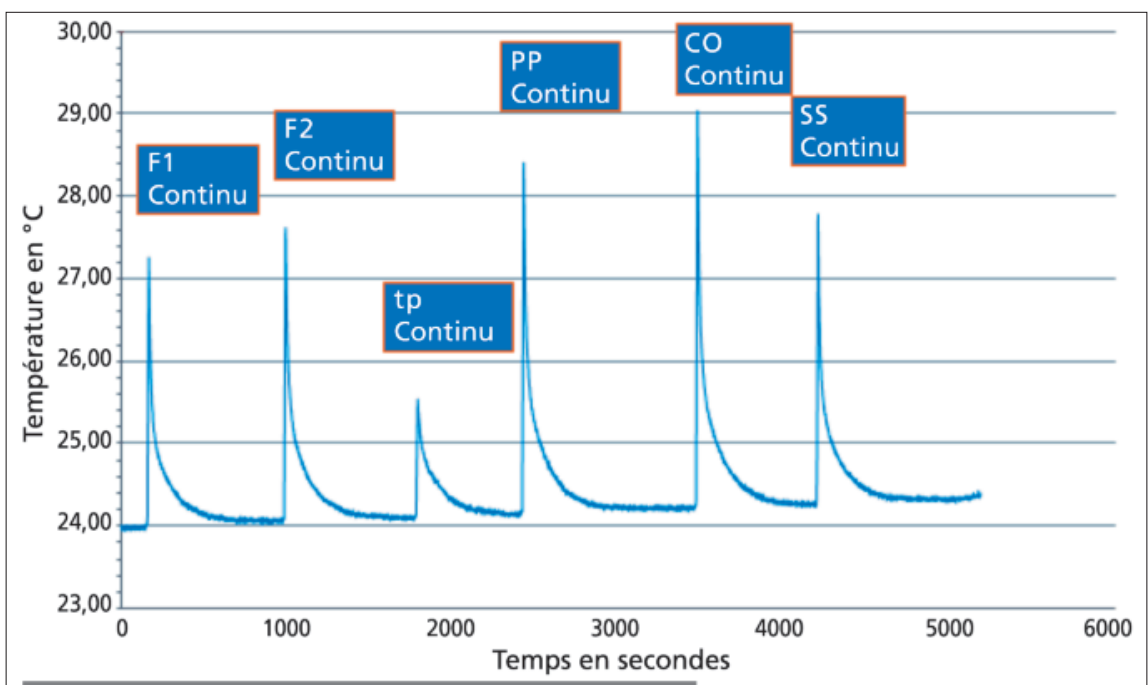

Mesure d'élévation de température durant l'insolation

Fig. 3 b Courbes d'élévation de température du prototype Scanwave en mode continu. 
ture. Le cahier des charges préconisé par le fabricant est respecté pour la limitation du phénomène d'élévation thermique.

\section{Détermination du retrait de polymérisation}

Les tests sont réalisés par l'intermédiaire de la technique du disque collé décrite comme la technique de Watts [5].

Le composite est placé entre une lame et une lamelle de microscope à l'intérieur d'un disque standardisé en cuivre.

Un capteur de déplacement (TESA GTL 21, précision 0,1 micromètre) est placé sur la lamelle et enregistre la rétraction au fur et à mesure de I'insolation à température ambiante (fig. 4). L'anneau en cuivre a une hauteur de $1,75 \mathrm{~mm}$ et un diamètre de $20 \mathrm{~mm}$. Le diamètre du matériau est calibré à $\approx 7 \mathrm{~mm}$. Le retrait de polymérisation linéaire est déterminé en fonction du temps.

Du fait des dimensions de l'anneau et du diamètre du matériau, ce retrait peut être considéré comme volumétrique [6]. Le composite

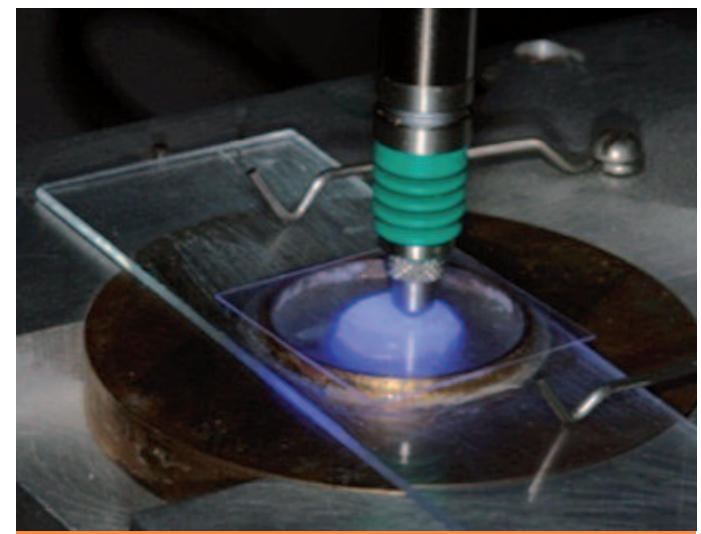

Fig. 4 Capteur de déplacement TESA durant la polymérisation.

Tetric Evoceram est utilisé en teinte B2. Le retrait est obtenu en millimètres (fig. 5) à rapporter à la hauteur de l'anneau, soit 1,75 millimètre.

Le retrait volumique au bout de 4 minutes est compris (tableau I) entre $1,1 \%$ et $1,13 \%$, sauf pour le mode TPC inférieur à $1 \%$.

La contraction de polymérisation mesurée est dépendante du montage utilisé [7].

Les mesures obtenues n'ont de valeur que comparative, pour les conditions définies dans cet




Tableau I Retrait de polymérisation $(\approx$ vol \%) par la technique de Watts pour différents modes du protopype Scanwave.

\begin{tabular}{|c|c|c|c|c|c|c|}
\hline \multicolumn{7}{c|}{ Teinte B2 Tetric Evoceram } \\
\hline \multicolumn{7}{|c|}{ Scanwave } \\
\hline Modes & F1C & F1P & tPC & PPC & COC & SCC \\
\hline Retrait polymérisation & $1,13 \%$ & $1,11 \%$ & $0,93 \%$ & $1,1 \%$ & $1,12 \%$ & $1,13 \%$ \\
(Moyenne \pm écart-type) & $\pm 0,02$ & $\pm 0,04$ & $\pm 0,06$ & $\pm 0,07$ & $\pm 0,04$ & $\pm 0,06$ \\
\hline
\end{tabular}

essai de type Watts. Le retrait de polymérisation (\% vol) après 1 heure, mesurée avec un dilatomètre à mercure est donné à 1,5\% par Ivoclar (dossier scientifique Tetric Evoceram ${ }^{\circledR}$ ). La contraction de polymérisation augmente avec l'augmentation des niveaux de densité d'énergie [8]. On retrouve cette relation avec le retrait minimal obtenu pour Scanwave en mode tP en continu.

\section{Détermination du degré de conversion}

Les tests sont réalisés par spectroscopie Raman (Aramis, Horiba Jobin) avec un laser héliumnéon qui émet à $632,8 \mathrm{~nm}$. Les échantillons sont préparés dans des moules en téflon de $5 \mathrm{~mm}$ de diamètre et de $5 \mathrm{~mm}$ d'épaisseur. Le composite est recouvert par un strip de mylar durant l'insolation pour éliminer la couche d'inhibition de l'oxygène de l'air. Les mesures sont réalisées avant polymérisation et après polymérisation par les différentes lampes. Les pics à $1640 \mathrm{~cm}^{-1}$ et $1610 \mathrm{~cm}^{-1}$ sont déterminés (fig. 6 a et b). Le degré de conversion est obtenu par la formule :

$D C \%=100 \times\left(1-R_{\text {polymérisé }} / R_{\text {non polymérisé }}\right)$

avec $R=\frac{\text { hauteur du pic à } 1640 \mathrm{~cm}^{-1}}{\text { hauteur du pic à } 1610 \mathrm{~cm}^{-1}}$

Le jaunissement apporté par la camphroquinone a nécessité pour les fabricants d'associer d'autres photo-initiateurs aux composites de type Bleach [9].

Pour tester cette possible influence sur la photopolymérisation [10], nous avons testé le degré de conversion de différentes teintes de composite Tetric Evoceram ${ }^{\circledR}$ soit, une teinte claire $A 1$, une teinte Bleach et une teinte foncée A3,5 (tableau II, III, IV).
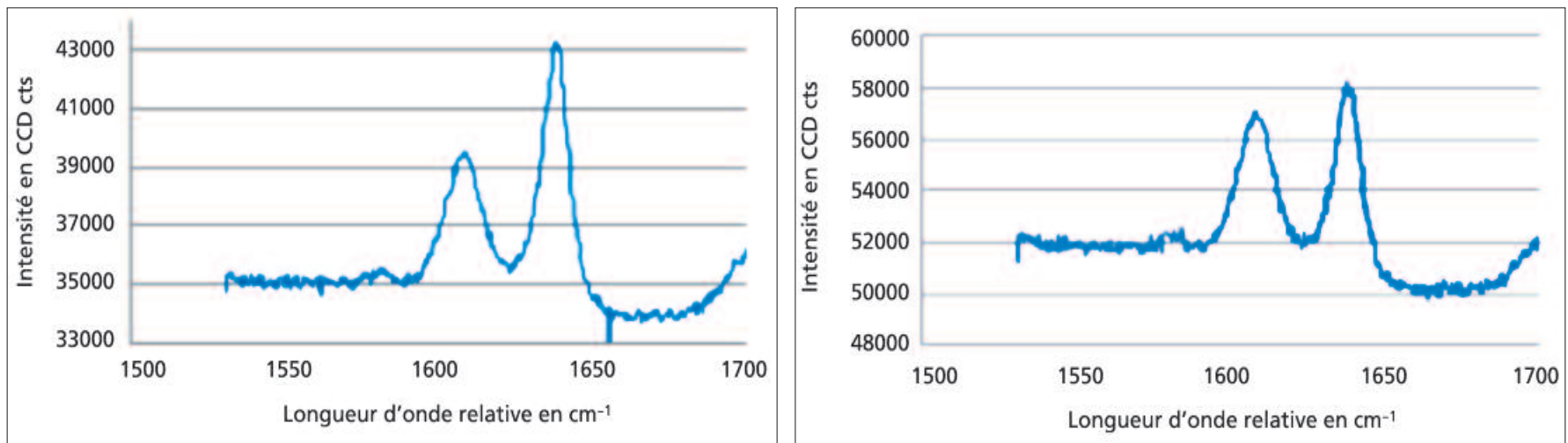

Fig. 6 a et b Pics Raman 1610 et $1640 \mathrm{~cm}^{-1}$ pour Tetric Evoceram ${ }^{\circledR}$ avant et après polymérisation. 


\begin{tabular}{|c|c|c|c|c|c|c|}
\hline \multicolumn{7}{|c|}{ Teinte A1 Tetric Evoceram } \\
\hline \multicolumn{7}{|c|}{ Scanwave } \\
\hline Modes & F1C & F1P & $t P C$ & PPC & $\mathrm{COC}$ & $\mathrm{scC}$ \\
\hline $\begin{array}{l}\text { Degré de conversion } \\
\text { (Moyenne } \pm \text { écart-type) }\end{array}$ & $\begin{array}{c}49,5 \% \\
\pm 6,4\end{array}$ & $\begin{array}{c}48,8 \% \\
\pm 3,7\end{array}$ & $\begin{array}{l}46 \% \\
\pm 8,2\end{array}$ & $\begin{array}{c}56,5 \% \\
\pm 12\end{array}$ & $\begin{array}{l}46,1 \% \\
\pm 0,7\end{array}$ & $\begin{array}{c}53,1 \% \\
\pm 3,9\end{array}$ \\
\hline
\end{tabular}

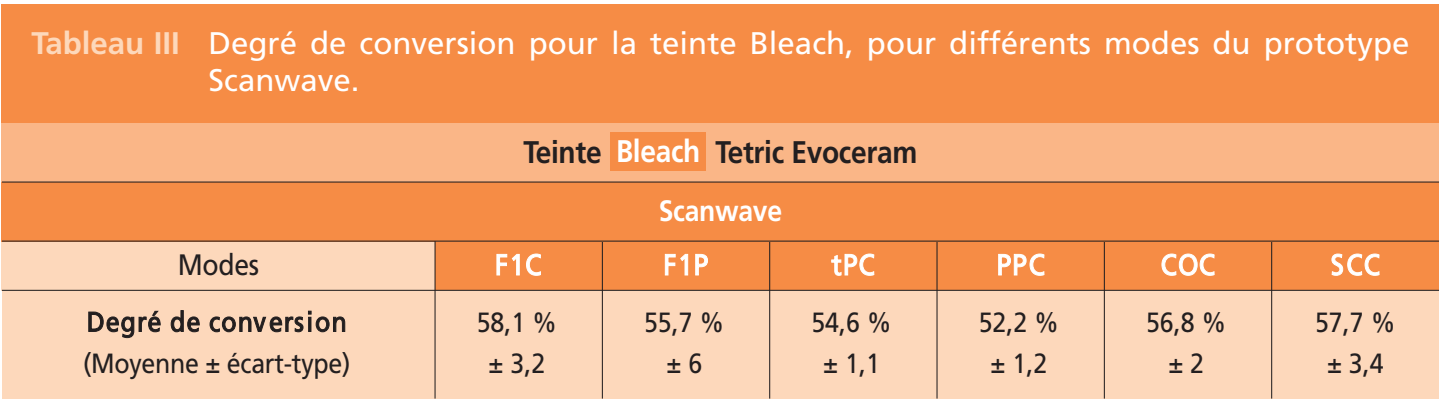

\begin{tabular}{|c|c|c|c|c|c|c|}
\hline \multicolumn{7}{|c|}{ Teinte $A 3,5$ Tetric Evoceram } \\
\hline \multicolumn{7}{|c|}{ Scanwave } \\
\hline Modes & F1C & F1P & tPC & PPC & $\mathrm{COC}$ & SCC \\
\hline $\begin{array}{l}\text { Degré de conversion } \\
\text { (Moyenne } \pm \text { écart-type) }\end{array}$ & $\begin{array}{l}59 \% \\
\pm 4,4\end{array}$ & $\begin{array}{c}55,9 \% \\
\pm 6,6\end{array}$ & $\begin{array}{c}47,2 \% \\
\pm 4,7\end{array}$ & $\begin{array}{c}41,8 \% \\
\pm 0,9\end{array}$ & $\begin{array}{c}42,2 \% \\
\pm 3,8\end{array}$ & $\begin{array}{r}45,2 \% \\
\pm 6,8\end{array}$ \\
\hline
\end{tabular}

Le degré de conversion obtenu est cohérent avec les autres études similaires. Miletic et Santini [11] donnent une valeur de 54,7 \% par spectroscopie Raman pour Tetric Evoceram ${ }^{\circledR}$ avec des échantillons non polis. Knežević et al. [12] obtiennent par spectroscopie FTIR des degrés de conversion compris entre 57 et $64 \%$ pour Tetric Evoceram ${ }^{\circledR}$.

On note des variations importantes pour une même teinte entre les différents modes de polymérisation, mais surtout entre les différentes teintes. Les variations dans la concentration des différents monomères mais aussi des différents initiateurs doivent expliquer ces résultats.
Détermination de la résistance au cisaillement de boitiers orthodontiques

Le but a été de comparer la lampe Scanwave avec une lampe de référence en orthodontie, Ortholux ${ }^{\mathrm{TM}}$ Luminous de $3 \mathrm{M}$ Espe.

Les modes de photopolymérisation sont :

- pour la lampe Scanwave, mode F1, 9 secondes ;

- pour la lampe Ortholuxтm, 9 secondes.

Un ciment de type CVIMAR (Fuji Ortho LC capsules, GC) et une résine de collage (Transbond XT, 3M Unitek) sont utilisés [13]. Des brackets 
Mini Uni-Twinтм 3M-Espe sont collés sur l'émail vestibulaire en respectant le protocole de collage du fabricant. Quinze dents sont testées par groupe.

Les tests sont réalisés par l'intermédiaire d'une machine de traction (fig. 7) dans un axe parallèle à la surface de collage avec une cellule M235-5DG (Physics Instrument). Les résultats sont traités sur le logiciel LabWiew5.

Les résultats ne montrent aucune différence significative (test de Friedman) entre la lampe Ortholuxтm de 3M Espe et le prototype Scanwave by MiniLED ${ }^{\circledR}$ de Acteon (tableau V).

Ces différentes expérimentations ainsi que celles de l'équipe de Birmingham (Adrian Shortall et William Palin) ont permis de confirmer l'efficience du concept élaboré par la société Acteon pour son prototype Scanwave by MiniLED ${ }^{\circledR}$.

Une réunion de travail à Mérignac s'est attachée à diminuer le nombre des modes de polymérisation et à les optimiser dans une optique d'applications en dentisterie restauratrice et en orthodontie.

La lampe Scanwave by MiniLED ${ }^{\circledR}$ sous sa forme définitive est commercialisée par la société Acteon depuis la fin de l'année 2011.

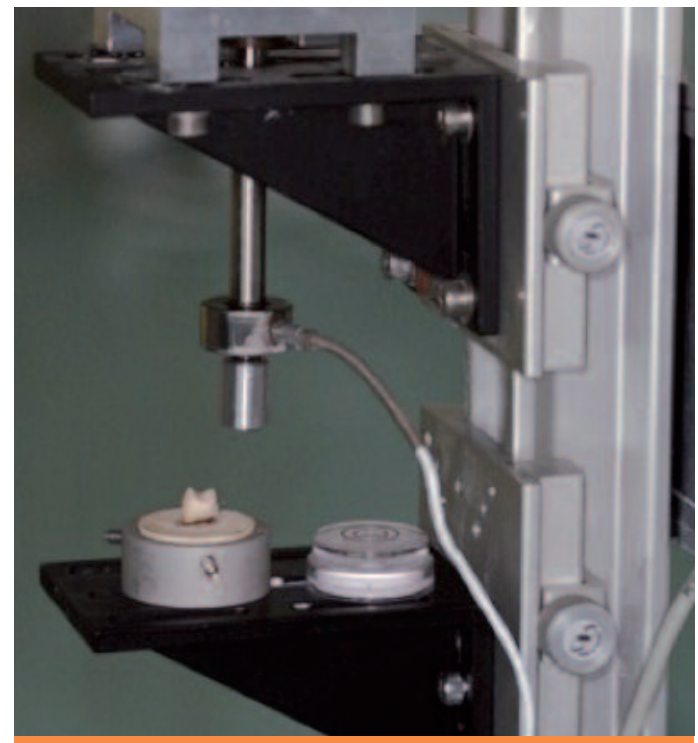

Fig. 7 Machine de traction avec la cellule M2355DG (Physics Instrument).

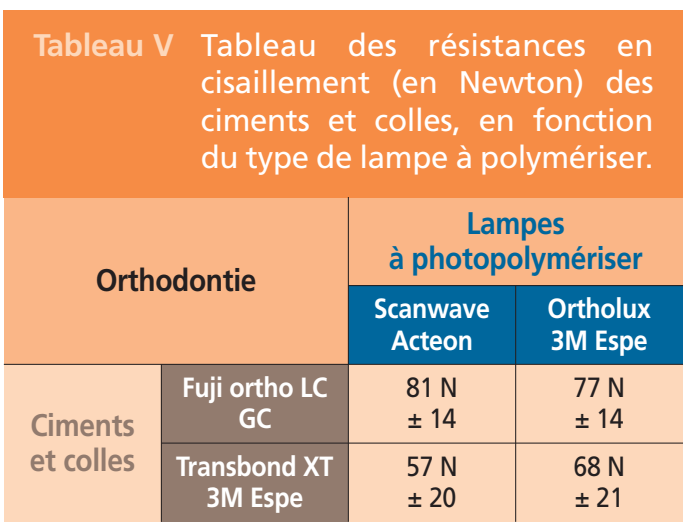

\section{Évaluation en clinique d'un nouveau type d'implant dentaire}

Les implants dentaires sont répertoriés en classe Ila dans la classification des dispositifs médicaux.

Les céramiques, dont la zircone, ont été proposées comme matériau alternatif au titane [14]. De plus, en cas d'intolérance au titane par le patient, la zircone est une très bonne alterna- tive [15]. Chez l'homme, très peu d'essais cliniques ont été menés et nous manquons d'études à long terme [16].

Le but de cette étude est d'évaluer et tester chez I'homme, la stabilité primaire et l'ostéointégration d'implants monoblocs en Zircone à l'aide du Periotest et d'indices cliniques parodontaux. 
Une étude chez le chien a été précédemment réalisée afin d'évaluer et d'analyser histologiquement l'ostéointégration des implants Zir-ROC Vissés ${ }^{\circledR}$ (fig. 8). Après une période de cicatrisation de 8 semaines, I'analyse histomorphométrique du contact os-implant est comparable à celle des études portant sur des implants en titane [17].

Pour notre essai clinique chez l'homme, les implants testés sont également de type ZIR-ROC Vissés ${ }^{\circledR}$, commercialisés par la société Paris Implants. Ces implants sont réalisés en zircone Y-TZP conformément à la norme NF ISO 13356 pour la réalisation d'implants chirurgicaux.

Le protocole chirurgical définit la mise en place dans les régions incisives, prémolaires et molaires, la référence employée étant le diamètre 4,25 $\mathrm{mm}$ pour lequel la longueur $\mathrm{du}$ corps endo-osseux pourra être de 10 ou $12 \mathrm{~mm}$. Un guide de pose personnalisé est systématiquement utilisé afin d'améliorer la standardisation du protocole. Ce guide correspond au système $2 \mathrm{INGIS}^{\circledR}$ QuickGuide, il est réalisé en titane-vanadium par fusion laser.

Le corps endo-osseux présente un cône d'étanchéité en direction coronaire dépourvu de spires, le corps de l'implant est entièrement fileté tandis qu'apicalement sont disposées des spires taraudantes. Le corps est surmonté d'un

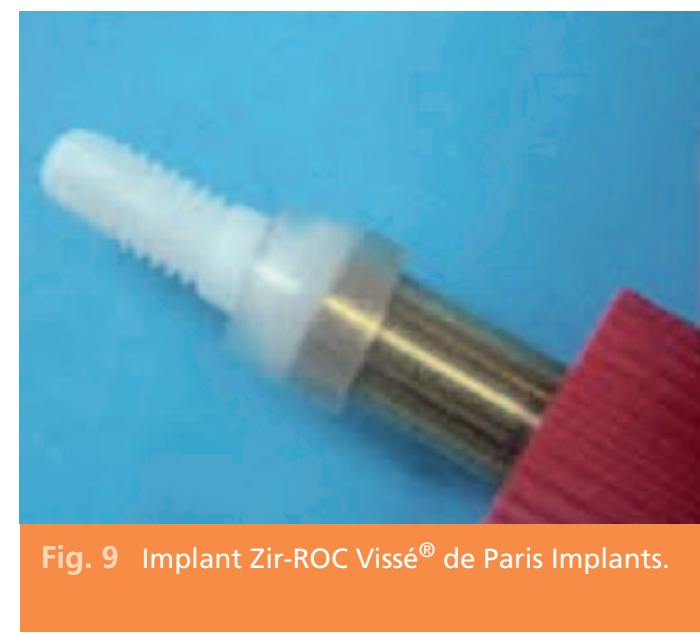



cône pour l'émergence transgingivale $(2,5 \mathrm{~mm})$ et d'un pilier à sa partie coronaire (fig. 9). Cet implant est disponible avec un pilier droit ou incliné de 0 ou 18 degrés. Pour cette étude, les angulations autorisées sont de 0 et 18 degrés.

L'étude est mono centrique pluri-investigateurs, comprenant deux étapes en ouvert et sans groupe contrôle. Le nombre de patients à inclure, corrélé au nombre moyen d'implants par patient (1,6 implant par patient), est de 27 à 33 patients pour atteindre la pose d'au moins 43 implants.

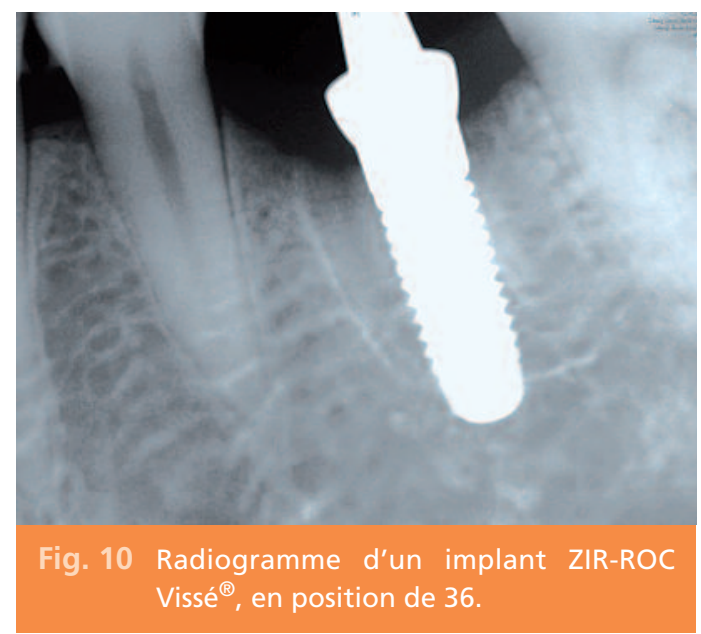


La durée de l'étude (suivi patient) est de deux ans. L'inclusion des patients est prévue sur un an.

L'étude a démarré en janvier 2012, et le premier implant a été placé (fig. 10).
Les objectifs de cette étude sont de mettre en évidence la bonne stabilité primaire des implants en zircone ZIR-ROC Vissés ${ }^{\circledR}$ de Paris Implants et leur bonne ostéo-intégration.

La fin de l'étude est prévue pour 2014.

\section{Conclusions générales}

Ces deux exemples d'évaluation de dispositifs médicaux s'inscrivent dans le cadre de la directive européenne 2007/47/CE transposée en droit français en mars 2010 [18].

La structure CED2M a été mise en place afin d'aider les fabricants du domaine dentaire à obtenir la déclaration CE de conformité qui atteste que leur produit est conforme aux exigences essentielles de santé et de sécurité et d'intégrer l'évaluation clinique dans la démonstration de conformité.

Différentes études sont en cours incluant des tests en laboratoire et des essais cliniques.

\section{Bibbliographie}

1. Conseil des Communautés Européennes.

Directive 93/42/CEE du conseil du 14 juin 1993 relative aux dispositifs médicaux. Journal Officiel des Communautés Européennes L 169 du 12.7.1993. http://eur-lex.europa.eu.

2. Leprince J, Devaux J, Mullier T, Vreven J, Leloup G.

Pulpal-temperature rise and polymerization efficiency of LED curing lights. Oper Dent 2010;35(2):220-30.

3. Atai M, Motevasselian F. Temperature rise and degree of photopolymerization conversion of nanocomposites and conventional dental composites.

Clin Oral Investig 2009;13(3):309-16.
4. Guiraldo RD, Consani S, Lympius T, Schneider LF, Sinhoreti MA, Correr-Sobrinho L. Influence of the light curing unit and thickness of residual dentin on generation of heat during composite photoactivation. J Oral Sci 2008;50(2):137-42.

5. Watts DC, Cash AJ. Determination of polymerization shrinkage kinetics in visible-light-cured materials: methods development. Dent Mater 1991;7(4):281-7.

6. Watts DC, Marouf AS. Optimal specimen geometry in bonded-disk shrinkage-strain measurements on light-cured biomaterials. Dent Mater 2000;16(6):447-51.
7. Tran CC, Angeletakis C, Shellard E. Dependence of bonded-disk shrinkage measurements on sample dimensions. IADR/AADR/CADR 80th General Session, San Diego, 2002.

8. Asmussen E, Peutzfeldt A. Polymerization contraction of resin composite vs. energy and power density of light-cure. Eur J Oral Sci 2005;113(5):417-21.

9. Ilie N, Hickel R. Can CQ be completely replaced by alternative initiators in dental adhesives? Dent Mater J 2008;27(2):221-8.

10. Schneider LF, Pfeifer CS, Consani S, Prahl SA, Ferracane JL. 
Influence of photoinitiator

type on the rate

of polymerization, degree

of conversion, hardness

and yellowing

of dental resin composites.

Dent Mater

2008;24(9):1169-77.

11. Miletic VJ, Santini A.

Remaining unreacted methacrylate groups in resin-based composite with respect to sample preparation and storing conditions using micro-Raman spectroscopy.

J Biomed Mater Res B Appl Biomater 2008;87(2):468-74.

12. Knežević A, Ristić $A$, Tarle $Z$, Pichler G, Musić S.

Degree of conversion and temperature increase during composite polymerisation with LED units of different intensity. Acta Stomatol Croat 2008;42(1):19-29.
13. Summers A, Kao E, Gilmore J, Gunel E, Ngan P. Comparison of bond strength between a conventional resin adhesive and a resin-modified glass ionomer adhesive: an in vitro and in vivo study. Am J Orthod Dentofacial Orthop 2004;126(2):200-6.

14. Andreiotelli M, Wenz HJ, Kohal RJ.

Are ceramic implants a viable alternative to titanium implants? A systematic literature review.

Clin Oral Implants Res 2009;20(Suppl 4):32-47.

15. Oliva $X$, Oliva J, Oliva JD. Full-mouth oral rehabilitation in a titanium allergy patient using zirconium oxide dental implants and zirconium oxide restorations. A case report from an ongoing clinical study. Eur J Esthet Dent 2010;5(2):190-203.
16. Wenz HJ, Bartsch J, Wolfart S,

Kern M.

Osseointegration

and clinical success

of zirconia dental implants:

a systematic review.

Int J Prosthodont

2008;21(1):27-36.

17 Khaldi L, Taleb-Bendiab C, Victoria-Herrera J, Torres JH,

Cuisinier F.

Ostéointégration

des implants

ZIR.ROC vissés ${ }^{\circledR}$

en zircone :

analyse histologique

chez le chien.

Stratégie Prothétique 2009;9(5):377-9.

18. JORF.

Rapport au Président

de la République relatif

à l'ordonnance $n^{\circ}$ 2010-250

du 11 mars 2010 relative

aux dispositifs médicaux. Journal Officiel $n^{\circ} 0060$ du 12 mars 2010.

\section{SUMMARY}

\section{Montpellier Medical Devices Evaluation Center (2MDEC). Laboratory and Clinical Applications}

\section{Jean-Christophe EGEA \\ Bruno PELISSIER \\ Alban DESOUTTER \\ Marie-Alix FAUROUX \\ Laurence POURREYRON \\ Frédéric CUISINIER \\ Bruno JACQUOT}

\section{Keywords \\ - medical device \\ - 93/42/EEC directive \\ - light curing \\ - dental implant}

European Directive 2007/47/EC is mandatory since March 21, 2010 for the entire European Union. A clinical evaluation is now required for all medical devices with enhanced regarding safety and performance.

To meet this need of assessment, Biohealth and Nanoscience laboratory established the Montpellier Medical Devices Evaluation Center (2MDEC).

Two assessments are carried out as part of 2MDEC.

The first evaluation was performed in the laboratory with a prototype LED light curing unit. Three standard parameters, correlated with the photopolymerization, were evaluated for applications in Restorative Dentistry and a shear strength test was performed for bonding cements used in orthodontics.

The second evaluation is in progress, in the University of Montpellier School of Dentistry clinics, on a new Zirconia implant. The clinical trial protocol is described. 